\title{
NOTAS
}

\section{ALGUNAS VARIANTES DE VERSOS DE HERRERA}

Arduo problema es el de fechar las distintas versiones de los poemas de Herrera, pero es preciso acometerlo, pues mientras no se resuelva no se podrá hablar con certeza de la evolución de su estilo ni, por consiguiente, de la evolución de la poesía y la lengua poética españolas. Durante tres siglos se ha prolongado el debate sobre las variantes, a veces en lorma encarnizada, y tanto los antiguos como los modernos ${ }^{1}$ han seguido un criterio más bien estético que científico. Falta todavía un estudio detenido de las variantes. Por otra parte, la discutidísima obra poética de Herrera sigue necesitando una edición esmerada. Para ambos propósitos ofrezco aquí algunos casos de identificación de variantes que hasta ahora se han desconocido o han quedado incompletamente explicados.

r) $\mathbf{H} /$ xxxirr $^{2}$ se encuentra en $\mathrm{P} / \mathrm{I} / \mathrm{XL}$ con dos variantes insignificantes

1 Me refiero a la acre respuesta de S. Batraglia, "Per il testo di Fernando de Herrera", FR, I (1954), núm. 1, 51-88, al artículo de J. M. BlecuA, "Los textos poéticos de Fernando de Herrera", $A O, 4$ (1954), 247-263.-La nota de A. Garrego Morele, "Una lanza por Pacheco", $R F E, 35$ (1951), 133-138, sí que atiende a los datos, o más bien a un dato que Battaglia cree bastante fidedigno (véase "Per il testo...", loe. eit., pp. 72-73), pero hay algunos reparos a propósito de este dato, que pienso comentar en otra ocasión.

${ }^{2}$ Cito con una sola inicial las siguientes ediciones: $\mathrm{A}=$ versos de Herrera incluidos en los preliminares o en las anotaciones de las Obras de Garcilasso de la Vega con Anotaciones de Fernando de Herrera, Sevilla, $1580 ; \mathrm{B}=$ Firnando dr. Herrera, Rimas inéditas, ed. de J. M. Blecua, Madrid, 1948; $\mathrm{C}=$ Fernando de Herrera, Contro. versia sobre sus Anotaciones a las obras de Garcilaso de la Vega. Poesias inéditas, So. ciedad de Bibliófilos Andaluces (prólogo firmado por José María Asensio), Sevilla, 1870; $\mathrm{H}=$ Algunas obras de Fernando de Herrera, Sevilla, 1582; $\mathrm{P}=$ Versos de Fer nando de Herrera, Strasbourg, 1916, ed. de Adolphe Coster (sigue el texto de Pacheco, Versos de Fernando de Herrera, / Emendados $i$ divididos por el / En tres libros, Sevilla, 1619). - Los números romanos en versales se refieren a alguno de los tres libros en que está dividida la edición $\mathrm{P}$; los números en versalitas se refieren, salvo indicación en contrario, a sonetos $\left(\mathrm{P} / \mathrm{I} / \mathrm{CxxI}\right.$ es el $121^{\circ}$ soneto del libro $1^{\circ}$ de $\mathrm{P} ; \mathrm{B} / \mathbf{x x x}$ es el $30^{\circ}$ soneto de la ed. de Blecua, etc.); para los poemas de Herrera incluidos en sus Anotaciones a Garcilaso en forma tan dispersa y fragmentaria, cito simplemente el número de página. - Sigo el sistema ortográfico de cada edición, excepto en el caso de la $i$ y en el de la diéresis. H, por ejemplo, no tiene punto en la $i$ y divide la diéresis poniendo un punto sobre la última vocal de la primera palabra y el otro sobre la primera vocal de la segunda; adopto el uso de $\mathrm{P}$, y pongo los dos puntos de la dićresis sobre la segunda vocal del hiato (para ärribar, etc.). En cuanto a las comillas y a los signos de admiración e interrogación, sigo en $\mathrm{P}$ el uso moderno, según la práctica de Coster. La puntuación de $\mathrm{B}$ es la de Blecua y no la del ms. 
de puntuación ya observadas por Coster; pero otra versión bastante distinta se halla también en $\mathrm{P} / \mathrm{I} / \mathrm{CxxI}$ :

\section{$\mathrm{P} / \mathrm{I} / \mathrm{CXXI}$}

Hebras, qu'Amor purpúra con el oro, en immortal ambrosia rociädo; tanto mi gloria sois i mi cuidado cuanto d'el solo sois mayor tesoro.

Vos, que los bellos astros i alto coro ornais, mis Luzes, d'esplendor sagrado, cuanto el impio es por vos mas estimado, tanto vos ónro umilde, i vos adoro.

Ardientes Rosas, Perlas d'Oriënte, Marfil vivo; i, angélica Armonia, cuanto vos míro mas, tanto m'inflamo.

I por vos cuanta pena l'alma siente; tanto es mayor valor i gloria mia; i tanto temo mas, cuanto mas amo.

\section{H/XXXIII}

Ardientes hebras, do s'ilustra el oro, de celestial ambrosia rociädo, tanto mi gloria sois i mi cuidado, cuanto sois del Amor mayor tesoro.

Luzes, qu'al estrellado i alto coro prestais el bello resplandor sagrado, cuanto es Amor por vos mas estimado, tanto umilmente os ónro mas i adoro.

Purpureas rosas, perlas d'Oriënte, marfil terso, i angélica armonia, cuanto os contémplo, tanto en vos m'in-

[flamo;

I cuanto pena l'alma por vos siente, tanto es mayor valor i gloria mia; i tanto os temo, cuanto mas os amo.

2) De la misma manera, Coster vio que $\mathrm{H} / \mathrm{XLI}$ y $\mathrm{P} / \mathrm{I} / \mathrm{CXI}$ son el mismo soneto, pero se le escapó otra variante, $\mathrm{P} / \mathrm{II} / \mathrm{Lxxv}$, que ofrece algunos cambios:

$$
\mathrm{H} / \mathrm{XLI}_{\mathrm{I}}
$$

Estoi pensando en $\mathrm{mi}$ dolor presente, i procúro remedio al mal instante; pero soi en mi bien tan inconstante, qu'a cualquier' ocasion buelvo la frente.

Cuando m'apárto, i pienso estar ausente, de mi peligro estoi menos distante, siempre voi con mis ierros adelante, sin que de tantos daños escarmiente.

Noble verguença del valor perdido, porque no abrasas este frio pecho, i deshazes mi ciego desvario?

Si tu me sacas deste error d'olvido; podrè dezir en onra deste hecho, que solo devo a ti poder ser mio.

\section{P/II/LXXV}

Pienso en mi pena atento i mal presente, i procúro algun medio al daño instante. pero soi en mi bien tan inconstante; que buelvo a la ocasion la incierta frente.

Cuando m'apárto i cúido estar ausente, menos de mi peligro estoi distante. voi siempre con mis culpas adelante, sin que de tantos ierros escarmiente.

Noble Verguença mia, qu'el perdido valor sientes, ¿porque no abrasa el pecho i vence tu virtud mi desvario?

Si d'el error i sombra d'el olvido me sacas, dirè'n onra d'este hecho; que solo devo a ti poder ser mio.

3) Coster notó la relación entre el soneto de $A$, p. 12g, y $\mathrm{P} / \mathrm{I} / \mathrm{xxx}$, y Blecua la de $\mathbf{P} / \mathbf{I} / \mathrm{xxx}$ con $\mathrm{B}$, p. 232, núm. 125; pero Blecua no menciona el soneto de $\mathrm{A}$, que ofrece variantes con respecto al poema de su manuscrito. $\mathrm{P} / \mathrm{I} / \mathbf{x x x}$ ofrece dos variantes de ortografía: v. 10 destilado (A, distilado) y v. 13 dessatado, evidente errata por desatado. Además, Coster repite en las notas el texto de $\mathbf{P}$ para el v. 11 , en vez de dar el texto de A, "ire 'n tu curso largo i estendido" (en P, "ire 'n tu luengo curso i estendido"). En B hay una errata: la versión del ms. empieza "Betys quen" [sic] Este tiempo solo y frio" en vez de "Qual Betis que este tiempo solo y frio". La variante del v. 6 de B ("dame que'en arbol uea de mi tormento / lamente trasformado") debe ser errata del copista del ms., en vez de la versión correcta de A y P ("arbol verde"). Fuera de estas erratas, coinciden B y A, excepto en el v. 3, donde B y $\mathrm{P}$ dicen "quieto mouimiento" frente a "callado movimiento" en A. 
4) Los vs. 1-2 de A, p. $14^{0}$ ("El ierto orrido risco, despeñado, / i la montana $\ddot{a}$ spera parece") se hallan sin variantes en $\mathrm{P} / \mathrm{III} / \mathrm{xxvn}$, vs. 9-10 ("El ierto, orrido risco, despeñado, / i la montaña äspera parece").

5) El v. 4 de A, p. 140 ("Dividen me de vos ô älma mia") es idéntico al v. 201 de B, elegía vi ("diuídenme de vos, o alma mía").

6) El v. 1 del soneto inédito publicado por Coster, "Poésies inédites de F. de Herrera", RHi, 42 (1918), p. 560 ("El oro crespo al Aura desparzido") se repite casi exactamente en $\mathrm{P} / \mathrm{I} / \mathrm{xcIx}, \mathrm{v}$. 1o ("el oro terso, a l'aura desparzido"); no se trata aquí de variante, sino de una curiosa autoimitación inconsciente por parte de Herrera.

7) Un trecho de 42 versos de A (desde la p. 321, v. 1, hasta la p. 322 , v. 22) corresponde a P/III, elegía 11, vs. 97-138, con estas variantes: "Solo puede nacer, i ser criado" (A, p. 322, v. 5) - "Puede solo nacer i ser criado" (P, v, 121); "ambos an d'acabar forçosamente" (A, p. 322, v. 12) - "ambos acabarán forçosamente" (P, v. 128); "a los regalos del amado Marte" (A, p. 322, v. 15) - "al regalo d'el dulce, amado Marte" (P, v. 131); "el uno a ötro dava mejor parte" (A, p. 322, v. 19) - "el uno dara'l otro mejor parte" (P, r. 135). Además, en A, p. 322, v. 9 , se lee crescimiento, frente a crecimiento en $P$, v. 125. La grafía crescimiento se aparta de las normas adoptadas por Herrera en otras partes de A (por ejemplo, crece, crecer, crecio, crecia en esta misma p. 322) y en $\mathrm{H}$ y $\mathrm{P}$, libros en que emplea su propio sistema ortográfico. (Otros ejemplos: $\mathrm{H} / \mathrm{xx} \mathrm{xv}, \mathrm{v} .3$, crecimiento; $\mathrm{H}$, elegía viI, v. 124 , creciente, etc., etc.).

8) El v. 1 de A, p. $55^{2}$ ("Cuando crece la sombra, i mengua el día") se encuentra en B, canción vir, v. 43 ("Quando creçe la sombra y mengua el día").

9) Los vs. 1-2 de A, p. 564 ("Fiero dolor, qu'el coraçon cuitado / tanto afliges i cansas, dolor fiero") se encuentran en $\mathrm{P} / \mathrm{MI} / \mathrm{xvI}$, vs. 1-2 ("Fiero dolor, qu'el coraçon cuitado / tanto afliges i cansas; dolor fiero").

10) $\mathrm{B} / \mathrm{xx}$ II corresponde a $\mathrm{P} / \mathrm{I} / \mathrm{xx}$, con alteraciones importantes:

Estaua en varios nudos recogido el cabello dorado a quien adoro; no cabello dorado, antes el oro, por quien alegre lleuo el mal sufrido.

Estaua el resplandor más encendido de aquellas luzes, del Amor tesoro, por quien mi gloria, ya perdida, lloro, pues son cavsa del daño a que e uenido.

La veste negra, la beldad del çielo era, y la voz de angélica armonía, el ayre y graçia, de diuino aliento.

Yo que buscaua triste algún consuelo, viendo el valor de aquesta Lumbre mía, llegué para lieuar mayor tormento.
Ardia, en varios cercos recogido, d'el crispante cabello en torno, el oro; qu'en bellos lazos coronado adoro, dichoso en el dolor d'el mal sufrido. Vibrava el esplendor esclarecido i dulces rayos, d'el Amor tesoro; por quien perdida búsco siempre, i lloro, la gloria de mi daño consentido.

Veste negra; descuido recatado; suäve voz d'angélica armonía era; mesura i trato soberano.

Yo, que tal no esperava, trasportado dixe'n la pura luz que m'encendia: "¡no encierra tal valor semblante umano!"

II) $\mathrm{B} / \mathrm{xxx}$ es variante de $\mathrm{B} / \mathrm{xLv}$. Los tercetos son iguales, salvo una errata del ms. en el v. 9 de $\mathbf{B} / \mathbf{x x x}$ (noble, en vez de nombre en $\mathbf{B} / \mathrm{xLvI}$ ) y un leve cambio de ortografía en el v. 10 (B/xxx, estanpado; $\mathbf{B} / \mathbf{X L v I}$, estampado). Pero los cuartetos son muy distintos: 


\section{$\mathrm{B} / \mathbf{x x x}$}

Vuestro süaue y tierno y noble canto, el espíritu eçelso y armonía, a mi pecho virtud çeleste embía y mueue en él furor diuino y santo.

Y si el Amor, cansado de mi llanto, diesse espaçio a la graue pena mía, en vuestra onrra la çítara alçaría, Moxcoso, avnque no igual la voz leuanto.

\section{B/XLVI}

¿Quál espíritu eçelso y noble canto pu[e]de'ençenderme más en su armonía que vuestro graue'estudio, que la uía enseña de virtud y de amor santo?

QQuántas veses, cansado de mi llanto, procuro terminar la pena mía, Moxcoso, y çelebrar como deuría vuestra honrra', a do el buclo no leuanto!

I2) E1 v. 14 de B/XLIV ("sino que consintáys que por vos pene") se repite en el 14 de P/III/LXXIv ("si no que consintais, que por vos péne"), pero en todos los demás versos son distintos estos sonetos.

I3) $\mathrm{B} / \mathrm{xLvIII}$ es igual a $\mathrm{P} / \mathrm{II} / \mathrm{LxvII}$, con variantes de interés. Creo que Espiritu, en el v. 9 de B, es errata del ms.; el plural de $\mathrm{P}$ tiene más sentido:

En sercos de oro fino y llama ardiente, de blancas rosas tiernas coronada, con hermosas figuras enlazada,

mi Luz vistió la pura y bella frente.

Los olores que esparse'el Orïente

y la'ámbar de sus hebras consagrada se mouieron con l'aura sossegada,

qual en las ondas nueuo sol luziente.

Espíritu de amor en aquel fuego armaron las saetas y cadena,

y Amor herido ardió y anudó el cuello.

Yo, preso y ençendido, quedé çiego, Conde, mas fue mayor mi graue pena, porque más me inflamé con el cabello.
En sortijas i flores d'oro ardiente, de perlas i rubies coronada, con hermosas figuras enlazada, cercó mi Luz la bella i blanca frente.

Los olores, que siembra el Oriënte, i l'ambar; qu'en sus hebras fue sagrada, se movieron con l'aura sossegada, cual en el manso mar el Sol luziente, Espíritus d'Amor en aquel fuego armaron las saetas i cadena, $i$, ardio el cruel herido, i preso el cuello.

Yo, traspassado el pecho, quedè ciego. mas fue mucho mayor mi acerba pena; qu'en llama eterna m'enredó el cabello.

\section{4) $\mathrm{B} / \mathrm{Lxx}$ y $\mathrm{P} / \mathrm{II} / \mathrm{CxI}$ son variantes:}

Renueuo al alma de mi error passado el tiempo que e perdido y el presente, ya que razón alguna no consiente que biua en esperanças engañado.

El cuello ya leuanto deslazado, que la señal del yugo impresa siente, y digo: “¿Quál de Amor graue açidente podrá lleuar la gloria de mi estado?"

Yo sé bien quánto duele vna esperança que huye, y vn temor que creçe'en pena, y quán vano es el fin de mi desseo.

Mas deshaze'esta simple confiança Amor, que al daño antiguo me condena, y alegre voy al mal que temo y ueo.
Pensoso buelvo a l'alma d'el passado tiempo el dolor, que tuve, i el presente, ya que razon alguna no consiente; qu'en dulce error padesca enagenado.

El cuello ya levánto deslazado; que la señal d'el yugo impressa siente. "¿cual tuyo, $\hat{o}$ impio Amor, grave acídente, digo, podrà mudar mi ufano estado?

Yo sè bien cuanto duele una esperança; que húye, $i$ un temor; que crece 'n pena, i cuan vano es el fin de mi desseo.

Mas deshazes, Cruel, mi confiança simple; qu'a tus engaños me condena, i voi alegre al mal, que temo i veo".

I5) De B/LXXI hay dos versiones en P: I/XLVII y II/XLIV, ya relacionadas éstas entre sí por Coster. Blecua no hace mención de $\mathrm{P} / \mathrm{I} / \mathrm{XLvII}$, que coincide casi perfectamente con B/LxxI, y esta omisión lo ha llevado a una corrección inteligente pero equivocada del v. 5 , que debe seguir a $\mathrm{P} / \mathrm{I} / \mathrm{xLvII}$, de que es copia, y no a $\mathrm{P} / \mathrm{II} / \mathrm{xLIv}$. El v. 5 dice: "Que sigo ausente, sin tu desvario" (P/I/XLVII) - "Sigo avsente, sin [bien], tu desuarío" (B/LXXI) - "Sigo ausente, sin bien, tu desvario" (P/II/XLIV); y el v. 14: "al' intimo llegò de l'alma mia" (P/I/XLVII) - "a lo íntimo 
llegó del alma mía" (B/LXXI) - "lo intimo penetrò de l'aima mia" (P/II/XLIV).

r6) Blecua omite el texto de un soneto del ms., fol. $233 \mathrm{v}^{0}$, por ser el mismo que $\mathrm{P} / \mathrm{I} / \mathrm{Cvi}$ (véase $\mathrm{B}, \mathrm{p} .233$, núm. 126). Hay otra variante, $\mathrm{P} / \mathrm{I} / \mathrm{L}$ n, en que no reparó Coster, y que difiere bastante de las otras dos. He aquí los textos de $\mathrm{P} / \mathrm{I} /$ LIII y de $\mathrm{P} / \mathrm{I} / \mathrm{CvI}$ :

Fueron d'un corto bien, que huye luego; antes que buelva la ocasion la frente, muestras, las qu'el Amor hallò presente; con que mi älma ardio en su eterno fuego.

Pero glorias d'un niño solo i ciego, que cedo las deshaze un acidente, ¿como pueden valer a un pecho ausente, qu'en su dolor no alcança algun sossiego?

Fundè mis esperanças en arena, qu'el viento esparze airado sin concierto. i rendido al temor perdi el recelo.

Cayeron, i el cruel por mayor pena en altas nubes desmayò desierto, ni alçar osando, n'inclinar el buelo.
Muestras de breve bien, que huye luego, antes que la ocasion buelva la frente, fueron las qu'el Amor hallò presente, con que mi álma ardio en su eterno fuego.

Pero glorias d'un niño solo i ciego, que presto las deshaze un acidente, ¿como pueden valer a un pecho ausente; que no sabe qu'es tiempo de sossiego?

Alcè mis esperanças sobre arena qu'el viento aparta, i lleva sin concierto, i no temo los golpes de mudança;

Cayeron, i el Amor, por mayor pena, quedò en las altas nuves descubierto; con temor, i sin fuerça, i confiança.

r7) Otro caso de texto con variantes es el de B/XIX y $\mathrm{P} / \mathrm{I} / \mathrm{LxxxI}$ (debo su conocimiento a la gentileza del propio José Manuel Blecua):

Los ojos leuanté yo, descuydado de mi futuro daño y cierta pena; el cuello suelto ya de la cadena que me traxo algún tiempo apremïado.

Y queriendo mirar (;ay duro hado!) el resplandor de aquella Luz serena, en quien Amor a fuego me condena, de quien con flechas tiene'el arco armado,

los suyos en los míos se'encontraron y luego con la fuerça de su fuego senti la dura flecha, el duro engaño.

Herido y çiego, ardiendo, me dexaron, y mi tormento en ellos se uio luego, con Amor conjurados en mi daño.
Alcé la vista a caso, descuidado de mi futuro afan i cierta pena, destexida d'el cuello la cadena; que me traxo en mil males enredado; I queriendo mirar (ai duro hado) el puro ardor d'aquella Luz serena; en quien Amor m'inflama i me condena, i con sus flechas vibra el arco armado,

Sus ojos en los mios encontraron, i con la fuerça de su fuego el pecho sintiò l'aguda vira en las entrañas.

Que no livianamente m'abrasaron, i el golpe fiero decendio derecho a mostrar en mi alma sus hazañas.

r8) El texto de $\mathrm{P} / \mathrm{I} / \mathrm{LII}$ se repite en $\mathrm{P} / \mathrm{I} / \mathrm{xcn}$; el v. 9 ofrece una variante: "Seguro podrè vêr d'oi mas la suerte" (P/I/LII) - "Seguro podrè vêr la indina suerte" ( $\mathrm{P} / \mathbf{I} / \mathrm{xcn})$; y el primer cuarteto presenta varias divergencias:

Kazon es ya, que la cansada vida, tanto tiempo sugeta 'I Amor vano, huya el fiero poder d'este tirano; i ya deslaze mi cerviz caida.
Iusto es, que la cansada, incierta vida, tiempo tanto sugeta 'I Amor vano, desdéñe 'I rigor impio; i d'el tirano yugo óse alçarse mi cerviz caida.

r9) Estos trozos de C, quintillas 11, vs. 66-80, 91-95, 111-115, 121-140, se hallan en serie consecutiva en $B A A E E$, t. 22, pp. $341 b-342 a$, donde faltan, además, las dos estrofas finales. Variantes: "lo que me deve doler" (C, v. 7o) - "lo que me debéis doler" (BAAEE, v. 5) (es preferible la lectura de C); "quanto mas cresce el tormento" (C, v. 8o) - "cuando más crece el tormento" (BAAEE, v. 15); "pueda" (C, v. 91) - "puede" 
( $B A A E E$, v. 16 ; errata, porque destruye la rima con queda); "truxeron" (C, v. 125) - "trajeron" ( $B A A E E$, v. 30 ).

2o) $\mathrm{C}$, redondillas $1 \mathrm{v}$, vs. $1-28,33-36,45^{-6}$, son las mismas de $B A A E E$, t. 22 , p. $341 a-b$, donde los trozos se publican también en forma consecutiva. Variantes: "que todo el mal olvidé" (C, v. 12) - "que todo el mundo olvidé" (BAAEE, v. 12) (la versión de $\mathrm{C}$ es quizá más herreriana, y encaja mejor con el resto del poema); "que vino ya, sin mas ver" (C, v. 15) - "que vivo ya, sin más ver" (BAAEE, v. 15, lectura ciertamente mejor); "mas por un hora que os veo" (C, v. 35) - "mas por una hora que os veo" ( $B A A E E$, v. 31) (para la variante un hora véase infra, núm. 21, la comparación entre $\mathrm{C}$, redondillas virI, vs. 1-2, y $B A A E E$, p. $34^{2}$, vs. 1-2); "ojos con cuia blandura" (C, v. 49) - "ojos en cuya blandura" (BAAEE, v. 41 ); "es fuerza que me hazeis / quando me mirais, mis ojos" (C, vs. 59-6o) - "es fuerza que me hacéis / cuando me miran mis ojos" (BAAEE, vs. $5^{1-52}$; será errata, porque el sujeto son siempre los ojos del v. 1 y del resto del poema).

2I) Otro caso en que $B A A E E$ presenta fragmentos del texto $\mathrm{C}$ como si fuesen un poema íntegro es el de $\mathrm{C}$, redondillas virI, vs. 1-12, 17-36, 45-68, y $B A A E E$, t. 22, p. 342a-b. J. M. Asensio pudo identificar los vs. 37-52 de C: "Más de una [ver] hemos buscado entre las obras publicadas de Herrera la composición de que formaron parte las cuatro redondillas, "Phaetón con ardor ciego.... que incluyó el poeta en sus Anotaciones a Garcilaso, pág. 136. No estaba tal poesía en ninguna de las colecciones, y la hemos encontrado entre las inéditas recogidas de Maldonado".. Si Asensio hubiera seguido con dos redondillas más, habría descubierto que ya Adolfo de Castro tenía publicada gran parte de esta poesía, aunque omitiendo los vs. 3744 de $\mathrm{C}$ y otros veinte. Castro también había visto la identidad de los últimos ocho versos de $A$, p. $13^{6}$ (y por lo tanto de $B A A E E$, pp. $337 b-338 a$, donde se imprimen los 16 de A) con $B A A E E$, p. $342 a$, vs. $33-4$ o; pero, fuera de una nota general del prólogo (p. vii), donde dice que va a presentar poesías publicadas en làs Anotaciones a Garcilaso, no da ninguna indicación sobre el origen de estos versos, y así los escondió de Asensio y aún de sí mismo, al parecer, pues no explica el hecho en su nota de la p. $342 a$. Además de los versos omitidos, hay algunas variantes: "Daua por ver una hora / serena i sin turbación" (C, vs. 1-2) - "Daba por veros un hora / serena y sin turbación" ( $B A A E E$, p. $34^{2}$, vs. 1-2); la lectura de $B A A E E$ parece una corrección equivocada; aunque Herrera no suele hacer hiato ante hora al final del verso, hay por lo menos un caso en B/xvn, v. 13: "que mi alma padeçe'en esta ora"; por otra parte, C tiene mucho más sentido, pues no es ella, sino él quien está intranquilo y triste (cl. vs. 9-10, "Confuso y aborrecido, / medroso y desesperado"). Otra variante: el v. 47 de C empieza "i atrevido...", lectura que aparece en A, p. 196, y se repite en $B A A E E$, p. $338 a$; en cambio, se suprime la conjunción, por errata, en $B A A E E$, p. $342 a$, v. 38 .

${ }^{3}$ C, Prólogo, pp. xxvi-xxvii. En la p. xxi y nota, dice Asensio que las poesías inéditas proceden de un códice de la Biblioteca Colombina. "Titúlase Obras de Fernando de Herrera, natural de Sevilla. Recojidas por $D$. Joseph Maldonado de Avila y Saavedra, Año 1637. (Ocupa el estante T, tabla 111, número 25)". 
Aunque no vale la pena estudiar la importancia ni la aplicación de estos casos sin tener en cuenta todas las demás variantes, quisiera indicar aquí unos ejemplos de la utilidad de las identificaciones hechas. Todos los casos de textos de A ofrecen un terminus ad quem de 1580 , y quizá las variantes ayuden a fechar partes de $\mathrm{A}$. La identificación de $\mathrm{A}$, p. 564 , vs. $1-2$, con $\mathrm{P} / \mathrm{III} / \mathrm{xvI}$, vs. $1-2$, nos revela los modelos de Herrera (Garcilaso, Virgilio y Ovidio) y su intento estilístico en estos versos de $\mathrm{P}$. Finalmente, un caso como la presencia de la palabra Conde en $\mathrm{B} / \mathrm{XIVIII}$ y su ausencia en $\mathrm{P} / \mathrm{II} / \mathrm{LXVII}$ nos da una razón más para relacionar los textos $\mathrm{B}$ y $\mathrm{H}$ frente al texto $\mathrm{P}^{4}$.

A. David Kossof

Brown University.

\title{
¿CINCO VERSILLOS QUE NO SON DE GÓNGORA?
}

Entre los diversos poemas atribuidos a Góngora por R. FoulchéDelbosc y Millé y Giménez ${ }^{1}$ figuran cinco versillos contra Lope de Vega, no exentos de cierta gracia:

\author{
Dicen que ha hecho Lopico \\ contra mí versos adversos; \\ mas si yo vuclvo mi pico, \\ con el pico de mis versos \\ a este Lopico lo-pico.
}

Pero esta quintilla figura a su vez en el ms. $3^{6} 57$ de la B. N. M., fol. 135 , atribuída a un "Juan Navarro de Cascante, poeta ridículo", con estas variantes:

\section{A LOPE DE VEGA}

Aunque tienc ese Lopico [contra mí] versos adversos, cuando yo extiendo mi pico, con el pico de mis versos a ese Lopico lo pico.

Claro está que esa sola mención no justificaría nada, pero es que junto a nuestro poemilla se copian otros muchos en los que se abusa de la misma técnica del jugueteo verbal, lo que prueba una misma mano. He aquí otro ejemplo:

\section{A Alarcón, poeta}

Con versos de corcovón

a Alarcón tanto le espanta

\footnotetext{
*Véase A. Costrr, Fernando de Herrera, Paris, 1908, pp. 192-194, donde habla de un fenómeno análogo de supresión en las variantes de $\mathrm{H}$ y $\mathrm{P}$.

1 Obras poeticas de don Luis de Cóngora, New York, 1921, t. 3. p. 42; Obras com. pletas de don Luis de Góngora, Madrid, 1932, p. 452. Véase en esta edición la nota de los hermanos Millé y Giménez, p. 1207.
} 